\title{
Modern Approaches to Language
}

\author{
Mark H. Bickhard \\ Lehigh University \\ mark@bickhard.name \\ http://www.bickhard.ws/
}

Recently, research on language has undergone major and accelerating transformations. This includes work addressing language related psychological processes and language development. At one point, such work was strongly dominated (at least in the United States) by Chomsky's theories and metatheories about language. This dominance has begun to fade as more fruitful models and approaches have been developed, with a special flowering of alternative approaches in the last decade or so. The intent of this special issue on modern approaches to language is to present a sampling of more recent approaches to understanding and studying language, with a particular emphasis on the relevance of these developments for psychologists.

Classic approaches treated language as a strongly formal system, with syntax specifying well formedness conditions on strings of abstract lexical items, semantics providing encoding meanings for those items, and pragmatics concerned with the uses to which such strings of items can be put. There was a brief flurry of excitement in the 1960s at the prospect that the formal syntactic rules for language strings might include psychologically real "transformations", but that hypothesis was quickly refuted. Since then, considerations of psychological reality, the actual dynamics of language and language processing, and the functional purposes and constraints involved in language interactions, productions, and understanding, have progressed, but have done so largely in the shadow of and against the pressures of a formalistic center of linguistics.

Nevertheless, dynamic and functional approaches have developed, and are now reaching into the core assumptions of the formalistic approach. These formalistic assumptions include:

- a base set of sound items that can serve as bricks in the construction of higher level items such as morphemes and words,

- rules for the formal combination of such formal items into well formed strings, 
- encoding rules for the meanings of those items,

- compositionality assumptions that the meanings of sentences are strictly compositional from the syntax and the encoded meanings of the items in that syntactic structure,

- and that language development honors the boundaries among syntax, semantics, and pragmatics, however much it may be the case that learning in one of these subdomains may be scaffolded by previous learning or innate scaffolds in a neighboring domain.

Recent movement has been away from these rather ad-hoc assumptions toward addressing and modeling much more realistic psychological processes - toward real language dynamics. In this issue, we present a strong sampling of some of these approaches - manifesting an interesting and important partial consilience regarding new ways of thinking about the nature of language and language processes.

If language is truly a dynamic process, both in the moment and over time, then grammar must emerge out of such dynamics. Among other consequences, such a dynamically emerging view of grammar would place both internal and external dynamical considerations at the center of considerations in accounting for grammatical structure. Hawkins (this issue) shows that grammatical regularities, considered across multiple languages, honor hierarchies of internal processing costs: he identifies multiple hierarchies of grammatical possibilities in which, for a given function, structural possibilities higher in the hierarchy demand greater processing costs from the individual. Language honors these processing cost hierarchies in the sense that, whenever a particular language accepts as grammatical any one level in such a hierarchy, it always accepts all lower processing cost possibilities as well. The processing cost hierarchies, then, impose universal constraints on the structure of the space of possible grammatically acceptable structures. Such influences of processing costs on grammatical structure are not consistent with formalist approaches to language.

On the external side, Diessel (this issue) notes that, if language is fundamentally a dynamic phenomenon, frequency can be expected to be an important influence on language - on use, acquisition, and on diachronic change. He illustrates the general 
point with numerous analyses addressing language acquisition, sentence processing, diachronic change, and some cross-linguistic tendencies. He concludes that "grammar is an emergent phenomenon that is fundamentally grounded in language use." This too is not consistent with a formalist approach.

Goldberg, Casenhiser, and White (this issue) address the processes by which we learn general linguistic categories from exposures to exemplars. They find that minimal exposure suffices, and that "generalizations in language are akin to non-linguistic generalizations". That is, the processes of generalizing on the basis of similarities seems to be similar to the learning of general non-linguistic categories - the processes of language learning do not seem to be language specific. A deeper theme in this work is the point that the learning of grammatical structures arises from category and organizational decompositions within general forms conveying particular forms of meaning. Grammatical structuring, then, does not arise as a formal system to which meanings can then be attached. Instead, grammar and meaning are progressively and only partially differentiated with respect to each other: the boundary between syntax and semantics is not a priori and is not strict - it is not formal.

If language is a social, dynamic process, then there is no apriori reason why the formal boundaries commonly postulated hold at all. Certainly this seems to be the case for the boundaries within what is taken as the domain of language studies, such as between syntax and semantics. But there is equally little reason to accept the strong boundary between formal cognitive aspects of language and social/emotional aspects of language. Greenspan and Shanker (this issue) outline an approach to language acquisition in which functional aspects of emotional signaling and emotional regulation between adult caregivers and infants plays a central, or even the central, role in language development. If language arises out of social interaction, rather than as an austere encoded transmission of formal mental contents, then emotional aspects must be involved.

One of the core foundational assumptions of classical language studies is that language units are constructed out of more basic units, which are constructed out of still more basic units, with the whole hierarchy bottoming out in little atoms of sound: 
phonemes. Port (this issue) argues that there are no psychologically real phenomena that answer to the notion of a phoneme. Phonemes are analytic idealizations and abstractions generated within the study of language, but not units or phenomena that are real in the actual processes of language production and understanding. Note that if this foundational level of linguistic bricks does not exist, then neither does any higher level of assumed linguistic atomic units, such as words. Phenomena that we attempt to address in such terms are dynamically emergent and partially differentiated for functional purposes. We take the partial functional differentiations as constituting fixed units only at the cost of ignoring the actual dynamics by which such phenomena are produced.

Finally, Bickhard (this issue) outlines a general dynamic model of language as a special kind of social interaction, illuminating several consequences of such an approach, and indicating how such a dynamic approach can potentially integrate points made in other papers. A major focus is on showing how the assumptions and arguments for the standard formal approach to language are invalid and unsound - there are no good reasons, either apriori or empirical, for maintaining the formal approach.

Most generally, all sciences, outside of studies of mental phenomena, have historically transcended substance and structural understandings of their subject matter to a recognition that they are fundamentally concerned with process, with dynamics. Phlogiston as a substance model of fire was replaced with combustion; caloric as a substance model of heat with random kinetic energy; and so on. That replacement, that move to process, is finally underway with respect to psychological phenomena, and, most especially, with respect to language.

\section{References}

Bickhard, M. H. (this issue). Language as an Interaction System. New Ideas in Psychology.

Diessel, H. (this issue). Frequency Effects in Language Acquisition, Language Use, and Diachronic Change. New Ideas in Psychology.

Goldberg, A. E., Casenhiser, D., White, T. R. (this issue). Constructions as Categories of Language. New Ideas in Psychology. 
Greenspan, S., Shanker, S. (this issue). The Developmental Pathways Leading to Pattern-Recognition, Joint Attention, Language, and Cognition. New Ideas in Psychology.

Hawkins, J. A. (this issue). Processing Typology and Why Psychologists Need to Know About It. New Ideas in Psychology.

Port, R. (this issue). Words, Symbols, and Rich Memory. New Ideas in Psychology. 